\title{
Small molecule non-peptide inhibitors of botulinum neurotoxin serotype $E$ : structure-activity relationship and a pharmacophore model
} \author{
Gyanendra Kumar ${ }^{a, b}$, Rakhi Agarwal ${ }^{a, c}$ and Subramanyam Swaminathan ${ }^{a^{*}}$ \\ ${ }^{a}$ Biology Department, Brookhaven National Laboratory, Upton, NY 11973, USA.

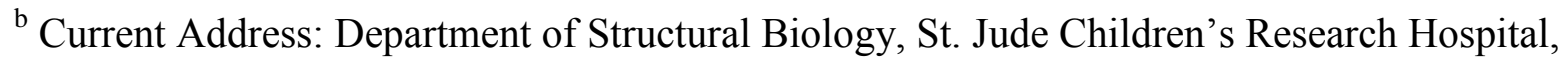 \\ Memphis, TN 38105 USA. \\ ${ }^{c}$ Current address: Chemistry Department, Nassau Community College, Garden City, NY 11530
} USA.

*Corresponding author: E-mail: swami@bnl.gov, Fax: (631) 344 3407; Tel: (631) 3443187.

Keywords: Botulinum neurotoxin, fluorene, inhibitor, structure-activity relationship, pharmacophore.

\begin{abstract}
Botulinum neurotoxins (BoNTs) are the most poisonous biological substance known to humans. They cause flaccid paralysis by blocking the release of acetylcholine at the neuromuscular junction. Here, we report number of small molecule non-peptide inhibitors of BoNT serotype E. The structure-activity relationship and a pharmacophore model are presented. Although non-peptidic in nature, these inhibitors mimic key features of the uncleavable substrate peptide Arg-Ile-Met-Glu (RIME) of the SNAP-25 protein. Among the compounds tested, most of the potent inhibitors bear a zinc-chelating moiety connected to a hydrophobic and aromatic moiety through a carboxyl or amide linker. All of them show low micromolar $\mathrm{IC}_{50}$ values.
\end{abstract}




\section{Introduction}

Botulinum neurotoxins (BoNTs) produced by various species of the genus Clostridium are classified as category A bioterrorism agents by the Centers for Disease Control and Prevention (CDC). With a lethal dose of $0.1-1 \mathrm{ng}$ per $\mathrm{kg}$ body weight, they are the most poisonous substances known so far. ${ }^{1}$ Their growing demand as therapeutic and cosmetic agents raises the risk of accidental overdose that may result in months of recovery for the patients at a heavy cost. ${ }^{2,3}$ The available antidotes against BoNTs are effective only against their extracellular fraction and not against symptom-causing fraction internalized by the neurons. ${ }^{4}$

BoNTs are grouped into seven different serotypes from A to $\mathrm{G}$ and further into dozens of isoforms. ${ }^{5}$ They are produced as a single inactive chain $(\sim 150 \mathrm{kDa})$ and secreted as active dichains containing a heavy $(\sim 100 \mathrm{kDa})$ and a light $(\sim 50 \mathrm{kDa})$ chain after proteolytic processing, held together by a conserved disulfide bond. ${ }^{6}$ The heavy chain itself consists of an N-terminal domain and a C-terminal domain ( 50 kDa each). The C-terminal domain of the heavy chain binds to specific receptors on the cholinergic neurons resulting in the internalization of toxin and the N-terminal domain facilitates the release of light chain into the cytosol. ${ }^{7,8}$ Recently, a host thioredoxin reductase has also been implicated in the release of light chain into the cytosol. ${ }^{9}$ The light chain is a zinc-dependent endopeptidase that cleaves one of the SNARE (Soluble Nethylmaleimide-sensitive factor Attachment protein REceptor) proteins and inactivates the SNARE complex. This disruption of SNARE assembly blocks the release of acetylcholine into the neuromuscular junction leading to flaccid paralysis. ${ }^{10}$ A beautiful schematic representation of the mode of action is given by Singh. ${ }^{11}$

The catalytic domain of various BoNT serotypes share $\sim 36 \%$ protein sequence identity and display specificity towards their substrate sequence. Serotype A and E cleave Synaptosomal- 
Associated Protein of $25 \mathrm{kDa}$ (SNAP-25), serotype C cleaves SNAP-25 and syntaxin, serotypes B, D, F and G cleave Vesicle-Associated Membrane Protein (VAMP). ${ }^{12-16}$ Since the symptoms of botulism appear only after the toxin has been internalized by the neurons, and has started disrupting the SNARE complex, the endopeptidase activity of the catalytic domain is an ideal drug target. Towards this goal, a number of substrate-based peptide inhibitors ${ }^{17-23}$ as well as small molecule non-peptide inhibitors ${ }^{24-32}$ have been identified against BoNT/A. Similarly, a few inhibitors targeting BoNT/B have also been identified. ${ }^{33,34}$ Among the three most common serotypes of BoNT - A, B and E, the last one has a unique domain organization. ${ }^{35}$ Remarkably, it also blocks the neurotransmission relatively faster compared to others probably due to the difference in organization (Fig. 1). ${ }^{36}$

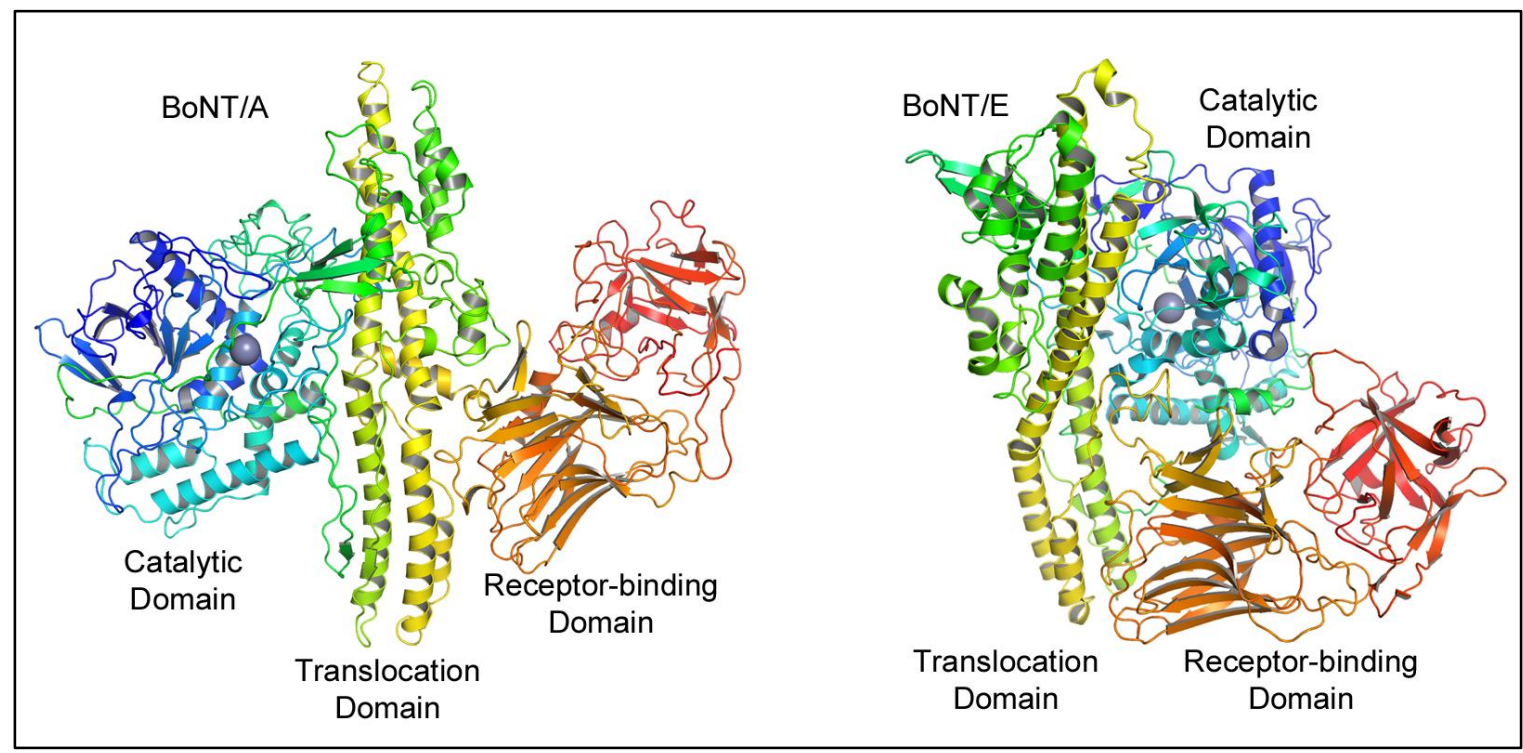

Figure 1. Domain organization in BoNT/A and BoNT/E. Structures are depicted in ribbons; rainbow colors, N-terminal is blue and C-terminal is red; zinc is show as grey sphere. BoNT/A created from PDB ID: 3BTA and BoNT/E from PDB ID: 3FFZ.

But, this serotype has not been studied extensively for inhibitor design and there are no known inhibitors for this serotype. Aided with the crystal structure of the catalytic domain of BoNT/E in its native form and in complex with a substrate-based peptide, ${ }^{37,38}$ we had conducted a virtual 
screening of NCI diversity set I and identified the first small molecule non-peptide inhibitor (SMNPI) - NSC77053 for this serotype recently. ${ }^{39}$ Herein, we report a number of novel SMNPIs of BoNT/E, along with the structure-activity relationship and a pharmacophore model for BoNT/E inhibition.

\section{Materials and methods}

Based on our most active compound NSC77053 (2(9H-fluorene-2-carbonyl) benzoic acid) identified previously ${ }^{39}$, a 2D similarity search was conducted against the NCI database of 250,250 compounds (https://cactus.nci.nih.gov/ncidb2.2/). Mostly we were looking for compounds with two parts similar to NSC77053 with different linkers and functional groups. The resulting 36 virtual 'hits' were obtained from the Drug Synthesis and Chemistry Branch, Developmental Therapeutics Program, Division of Cancer Treatment and Diagnosis, National Cancer Institute. They were tested against the purified BoNT/E catalytic domain to determine their inhibition of the endopeptidase activity in an HPLC-based assay. These compounds were $>90 \%$ pure based on LC/mass spectrometry as per the supplier.

\subsection{Docking simulations}

The docking program AutoDock 4.2 was used to calculate the binding modes and binding energies of the compounds against BoNT/E. The atomic coordinates from the crystal structure of BoNT/E catalytic domain (pdb id: $3 \mathrm{D} 3 \mathrm{X})^{38}$ were used as the receptor after adding hydrogens and assigning atomic charges according to Merck Molecular Force Field (MMFF94x) as

implemented in the Molecular Operating Environment (MOE 2011.10). ${ }^{40}$ The energy-minimized atomic coordinates of small molecules were generated using the Builder module of MOE, with the MMFF94x force field. The conformational flexibilities in these molecules were defined by 
using AutoTors utility provided with AutoDockTools. The AutoGrid program was used to generate $3 \mathrm{D}$ grid maps of the active site for each atom type of the compounds. Hydrogen bonds and van der Waals interactions were modeled using the Lennard-Jones parameters 12-10 and 126 incorporated in the AutoDock suite, and the distance dependent dielectric permittivity of Mehler and Solmajer ${ }^{41}$ was used for calculation of electrostatic grid maps. The Lamarckian genetic algorithm with pseudo Solis and Wets modification (LGA/pSW) method was used with default parameters. For each small molecule, 20 docking runs were performed and cluster analysis was conducted for the docked conformations with an rms tolerance of $2 \AA$.

\subsection{Inhibition assay}

Inhibition assays were conducted by following the previously published method. ${ }^{38}$ Since we selected only 36 hit compounds we did not rank them according to energy and conducted inhibition assays on all of them. From our experience we found that there is no direct correlation between the binding energies and the experimental $\mathrm{IC}_{50 .}{ }^{39}$ We determined $\mathrm{IC}_{50}$ values for the top best compounds using a cutoff value of $50 \%$ inhibition for $20 \mu \mathrm{M}$ inhibitor

concentration. $20 \mu \mathrm{M}$ concentration of inhibitor was chosen based on our previous experience. ${ }^{39}$ Briefly,

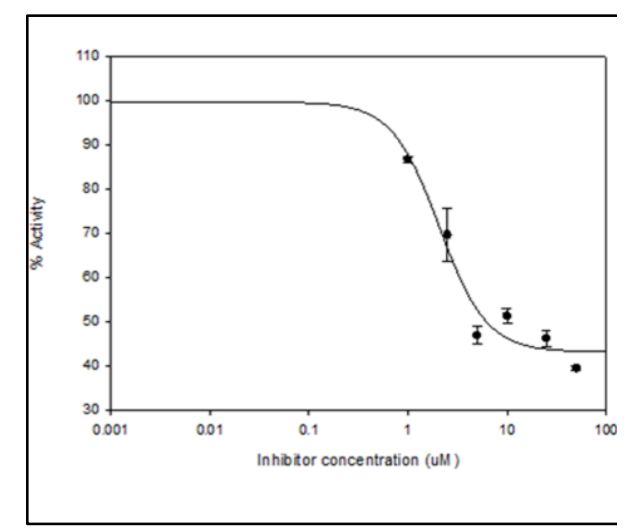

Figure 2. A representative dose response plot for NSC132252. Measurements were in triplicate. The plot was generated using Sigmaplot $^{\circledR}$.

the reactions were carried out in $30 \mu \mathrm{L}$ of reaction mixture containing $20 \mathrm{mM}$ HEPES buffer, $\mathrm{pH}$ 7.4, $2 \mathrm{mM}$ DTT, $10 \mu \mathrm{M}$ zinc acetate, with $0.5 \mathrm{nM}$ BoNT/E light chain and $118 \mu \mathrm{M}$ substrate peptide SNAP-25 (residues 146-186). The reaction mixtures were incubated at $37^{\circ} \mathrm{C}$ to achieve $<20 \%$ substrate to product conversion. The reaction was stopped by adding $3 \mu \mathrm{L}$ of $10 \%$ 
trifluoroacetic acid. The samples underwent HPLC analysis (Beckman Coulter) in a Discovery Bio-Wide pore C18 column with acetonitrile and aqueous phase gradient (Buffer A: 0.1\% trifluoroacetic acid in water; Buffer B: $90 \%$ acetonitrile, 0.1 trifluoroacetic acid). The amount of cleaved product was calculated by using the $32 \mathrm{Karat}^{\mathrm{TM}}$ program provided by Beckman Coulter. For the inhibition assay $1,2.5,5,10,25$ and $50 \mu \mathrm{M}$ concentration of inhibitors were used. We used our previously identified compound NSC77053 and DMSO as control. The data were analyzed, and $\mathrm{IC}_{50}$ values were calculated by using Graph-Pad software. A representative dose dependent inhibition plot is shown for NSC132252 (Fig. 2). Experiments were conducted in triplicate.

\subsection{Pharmacophore modeling}

The pharmacophore model was elucidated by using the molecular modeling program MOE 2011.10. Flexible alignment of the active compounds was conducted to superimpose them and identify the most common and conserved pharmacophore features.

\section{Results and discussion}

We tested a number of new compounds based on our first hit compound NSC77053 (2(9H-fluorene-2-carbonyl) benzoic acid) ${ }^{39}$ and identified a related new lead compound that inhibits BoNT/E endopeptidase activity with an $\mathrm{IC}_{50}$ of $2.4 \mu \mathrm{M}$. The structure of $\mathbf{N S C 7 7 0 5 3}$ could be divided into two distinct parts - a benzoic acid moiety and a fluorene moiety connected through a carbonyl linker (Fig. 3). According to our docking simulations, this compound mimics several interactions observed in the co-crystal structure of the RIME-BoNT/E complex. ${ }^{38}$ The carboxyl group of the benzoic acid moiety coordinates with the zinc and forms a hydrogen bond with the side chain $\mathrm{OH}$ of Tyr350. The benzene ring occupies the $\mathrm{S} 1$ ' site that corresponds to the 
binding site of isoleucine of the peptide RIME of substrate protein SNAP-25. The

aromatic/hydrophobic fluorene moiety occupies the S2' and S3' sites where the hydrophobic side-chain of methionine of the substrate peptide RIME binds. ${ }^{39}$ In order to deduce the structure-activity relationship for the SMNPIs of BoNT/E, we analyzed many diverse functional groups on both of these distinct parts (Fig. 3). We selected and tested thirty-six different compounds representing

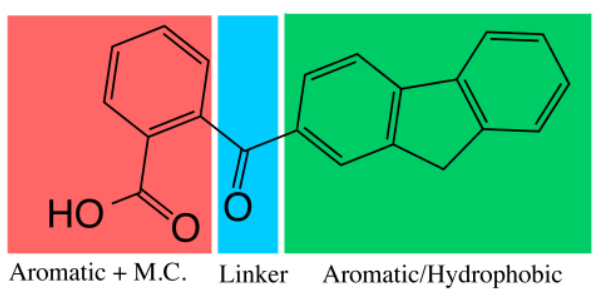

Figure 3. The 3-part initial pharmacophore model from NSC77053 that was used to screen the NCI compound database to identify potential inhibitors of BoNT/E. M.C. indicates metal chelator.

variations on either part and identified five new compounds with $\mathrm{IC}_{50}$ values in low micromolar range. Four of these compounds have a fluorene moiety and the fifth one has an anthracene moiety in its aromatic/hydrophobic part. While the original compound NSC77053 had a carbonyl linker connecting the two parts, the new fluorene containing inhibitors have an amide linker.

\subsection{Structure-activity relationship}

The compounds were initially tested at $20 \mu \mathrm{M}$ concentration and the $\mathrm{IC}_{50}$ values were determined for those showing more than $50 \%$ inhibition at this concentration. The structures of these compounds and their corresponding inhibitory values are provided in Tables 1 and 2 . The most potent compound NSC75271 (3-hydroxy-N-phenyl-2-anthracenecarboxamide) inhibited BoNT/E endopeptidase activity with an $\mathrm{IC}_{50}$ of $2.4 \mu \mathrm{M}$ (Table 1 ), and shows a similar mode of binding as predicted earlier for NSC77053 according to docking simulations. The anthracene moiety binds in the same S2' pocket as the fluorene moiety of NSC77053, but the phenyl group occupies S1 pocket instead of S1' pocket. In the absence of a carboxylic group to chelate the zinc 
ion, it is the $\mathrm{C}=\mathrm{O}$ group of the linker that chelates the zinc ion (Fig. 4).$^{39}$ Of the four fluorene compounds, three have comparable $\mathrm{IC}_{50}$ values at $\sim 3 \mu \mathrm{M}$ :

NSC132241 (2'-((9H-fluoren-2ylamino)carbonyl)-4,4'bis(hydroxy(oxido)amino)[1,1'-biphenyl]-

2-carboxylic acid), NSC132252 (4,4'dichloro-2'-(((5,7-dichloro-9H-fluoren-2yl)amino)carbonyl)[1,1'-biphenyl]-2-

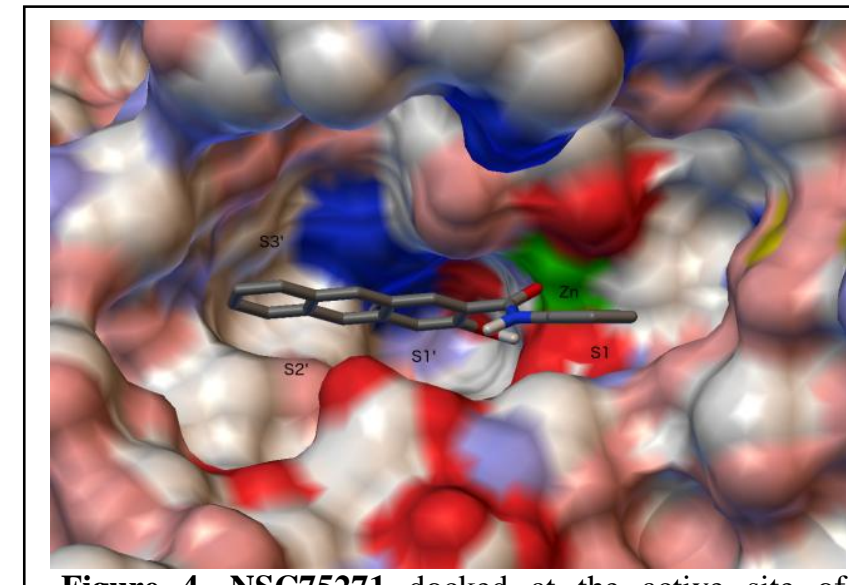

Figure 4. NSC75271 docked at the active site of BoNT/E. The inhibitor is shown in stick model with element colors. BoNT/E is displayed with molecular surface in the following color scheme: neutral oxygen and nitrogen atoms are pink and light blue, respectively, charged oxygen and nitrogen atoms are red and dark blue, respectively, zinc is green.

carboxylic acid) and NSC132249 (4,4'-dichloro-2'-((9H-fluoren-2-ylamino)carbonyl)[1,1'biphenyl]-2-carboxylic acid) with $\mathrm{IC}_{50}$ of $2.7,3.0$ and $3.2 \mu \mathrm{M}$, respectively (Table 1).

Presumably, the lack of a zinc-chelating carboxylic group and torsional rigidity are the reasons for a reduced inhibitory activity of NSC132253 (3,9-dichloro-6-(5,7-dichloro-9H-fluoren-2-yl)$5 \mathrm{H}$-dibenzo[c,e]azepine-5,7(6H)-dione), as it shows an $\mathrm{IC}_{50}$ of $9.8 \mu \mathrm{M}$. Overall, a common feature of the inhibitors of this class is the presence of a fluorene moiety and a new type of zincchelator linked through an amide group. Addition of two chlorines on the fluorene moiety improves the inhibition activity slightly (as seen in NSC132252 vs NSC132249, and NSC132253 vs NSC132250). On the other hand, addition of a $\mathrm{C}=\mathrm{O}$ or $\mathrm{OH}$ group has led to decrease in inhibitory activity by half (NSC67690 vs NSC67692 or NSC127134) (Table 1).

\subsection{Importance of the fluorene moiety}

Keeping the benzoic acid moiety constant, we tested a number of alternatives for the fluorene moiety (Table 2). Surprisingly, all of them led to a decrease in inhibitory potency, 
showing that a fused three-ring moiety is preferred. Replacing the fluorene moiety with a tworing system has resulted in a substantial decrease in inhibitory potency (NSC135940 and

NSC75270) (Table 2). Replacing this aromatic/hydrophobic moiety with a single ring (NSC6646) or addition of hydrophilic groups (NSC518963 and NSC5003) reduces the inhibitory activity of the compounds, indicating the importance of aromatic/hydrophobic group (Table 2). Interestingly, the fluorene group alone has no substantial effect on BoNT/E inhibition (only $17.3 \%$ inhibition at $20 \mu \mathrm{M}$ concentration, NSC6787) but when linked with a possible zincchelating group, the inhibitory activity is substantially enhanced (80\% inhibition at $20 \mu \mathrm{M}$, NSC77053) (Table 2).

\subsection{A pharmacophore model for BoNT/E inhibition}

Out of the seven serotypes of BoNT, pharmacophore models have been developed only for serotype A so far. For BoNT/A, the consensus is on the requirement of a metal chelator and two positively charged groups on either side of the metal chelator, although as many as seven different pharmacophore features have been proposed based on BoNT/A inhibitors identified so far. To develop a pharmacophore model for the inhibition of BoNT/E, we conducted flexible

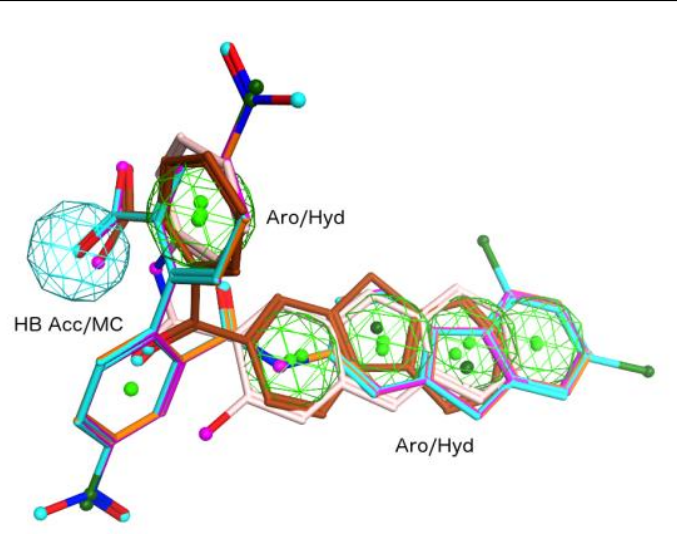

Figure 5. A pharmacophore model for BoNT/E inhibition. Inhibitors are shown in stick model with the following color scheme - NSC77053 (brown); NSC75271 (pink); NSC132241 (orange); NSC132252 (cyan); and NSC132249 (purple). Pharmacophore features are shown in mesh, green mesh indicates an aromatic/hydrophobic feature, and cyan mesh indicates a hydrogen bond acceptor/metal chelator.

alignment of the hit compounds using MOE. The molecules superimposed very well according to their pharmacophore features. The common pharmacophore features among all the hit 
compounds NSC77053, NSC75271, NSC132241, NSC132252 and NSC132249 are - (i) the three-ring aromatic/hydrophobic feature exemplified by a fluorene or an anthracene moiety; (ii) another aromatic/hydrophobic feature exemplified by a single phenyl ring feature as seen in the hit compounds that is connected to the three-ring system either through an amide linker or a carboxy-linker and (iii) a hydrogen bond acceptor or metal chelator that possibly participates in chelating zinc (Fig. 5). While metal chelation is a common pharmacophore feature between serotypes $\mathrm{A}$ and $\mathrm{E}$, the other pharmacophore features are distinct. This is not surprising because the cognate substrates for BoNT/A and BoNT/E are different. While BoNT/A substrate sequence for $\mathrm{P} 1-\mathrm{P} 1$ '-P2'-P3' stretch is QRAT, that for BoNT/E is RIME. In their S1' pocket, BoNT/A prefers a positively charged group, like the guanidinium side chain of Arginine but BoNT/E prefers a hydrophobic moiety i.e. the side chain of Isoleucine. On the basis of certain BoNT/A inhibitors, planar zone 1 and zone 2 have been proposed for BoNT/A previously. ${ }^{42}$ On the basis of BoNT/E inhibitors discussed in this article, we believe this pharmacophore feature may also overlap between the two serotypes as the aromatic features required in BoNT/E inhibitors fulfill this criterion. So, a common pharmacophore model applicable to more than one serotype of BoNT seems feasible.

\section{Conclusion}

Four new SMNPIs of BoNT/E have been identified and the structure-activity relationship provides insight into the inhibition of BoNT/E by SMNPIs. We also have developed the first pharmacophore model for the inhibition of BoNT/E. This pharmacophore model has some unique features for BoNT/E inhibition and some common features with BoNT/A. It could be used for virtual screening of compound libraries to identify novel BoNT/E inhibitors from different classes of compounds. 


\section{Acknowledgements}

Research was supported by an award from DTRA BO742081 under DOE prime contract No. DEAC02-98CH10886 with Brookhaven National Laboratory. The United States

Government retains and the publisher, by accepting the article for publication, acknowledges that the United States Government retains a non-exclusive, paid-up, irrevocable, world-wide license to publish or reproduce the published form of this manuscript, or allow others to do so, for United States Government purposes.

\section{Figure legends:}

Figure 1. Domain organization in BoNT/A and BoNT/E. Structures are depicted in ribbons; rainbow colors, $\mathrm{N}$-terminal is blue and $\mathrm{C}$-terminal is red; zinc is show as grey sphere. BoNT/A created from PDB ID: 3BTA and BoNT/E from PDB ID: 3FFZ.

Figure 2. A representative dose response plot for NSC132252. Measurements were in triplicate. The plot was generated using Sigmaplot ${ }^{\circledR}$.

Figure 3. The 3-part initial pharmacophore model from NSC77053 that was used to screen the NCI compound database to identify potential inhibitors of BoNT/E. M.C. indicates metal chelator.

Figure 4. NSC75271 docked at the active site of BoNT/E. The inhibitor is shown in stick model with element colors. BoNT/E is displayed with molecular surface in the following color scheme: 
neutral oxygen and nitrogen atoms are pink and light blue, respectively, charged oxygen and nitrogen atoms are red and dark blue, respectively, zinc is green.

Figure 5. A pharmacophore model for BoNT/E inhibition. Inhibitors are shown in stick model with the following color scheme - NSC77053 (brown); NSC75271 (pink); NSC132241 (orange); NSC132252 (cyan); and NSC132249 (purple). Pharmacophore features are shown in mesh, green mesh indicates an aromatic/hydrophobic feature, and cyan mesh indicates a hydrogen bond acceptor/metal chelator. 
Table 1. Novel anthracene and fluorene inhibitors of BoNT/E

\begin{tabular}{|c|c|c|c|c|}
\hline S. No. & NSC No. & Structure & $\begin{array}{c}\text { \% Inhibition } \\
\text { at } 20 \mu \mathrm{M}\end{array}$ & $\begin{array}{l}\mathrm{IC}_{50} \\
\boldsymbol{\mu \mathbf { M } ^ { * }}\end{array}$ \\
\hline 1 & 75271 & & 70.1 & $2.4 \pm 0.4$ \\
\hline 2 & 132241 & & 82.0 & $2.7 \pm 1.9$ \\
\hline 3 & 132252 & & 79.1 & $3.0 \pm 6.7$ \\
\hline 4 & 132249 & & 88.3 & $3.2 \pm$ n. a \\
\hline 5 & 132253 & & 68.9 & $9.8 \pm 4.4$ \\
\hline 6 & 132250 & & 48.0 & ND \\
\hline 7 & 128598 & & 47.6 & ND \\
\hline 8 & 67690 & & 37.2 & ND \\
\hline 9 & 132251 & & 24.7 & ND \\
\hline
\end{tabular}




(2)

*The experiments were conducted in triplicates except for NSC132249. ND: not determined.

N.a. not available. 
Table 2. NSC77053 and its analogues tested against BoNT/E

S. No. NSC No.




\section{References:}

1. Schiavo, G.; Rossetto, O.; Benfenati, F.; Poulain, B.; Montecucco, C. Tetanus and botulinum neurotoxins are zinc proteases specific for components of the neuroexocytosis apparatus, Ann. New York Acad. Sci. 1994, 710, 65-75.

2. Souayah, N.; Karim, H.; Kamin, S. S.; McArdle, J.; Marcus, S. Severe botulism after focal injection of botulinum toxin, Neurology 2006, 67, 1855-1856.

3. Wein, L. M.; Liu, Y. Analyzing a bioterror attack on the food supply: the case of botulinum toxin in milk, Proc. Natl. Acad. Sci. U.S.A. 2005, 102, 9984-9989.

4. Cai, S.; Singh, B. R. Strategies to design inhibitors of Clostridium botulinum neurotoxins, Infectious disorders drug targets 2007, 7, 47-57.

5. Hill, K. K.; Smith, T. J. Genetic diversity within Clostridium botulinum serotypes, botulinum neurotoxin gene clusters and toxin subtypes, Curr. Top. Microbiol. Immunol. 2013, 364, $1-20$.

6. Sathyamoorthy, V.; DasGupta, B. R. Separation, purification, partial characterization and comparison of the heavy and light chains of botulinum neurotoxin types A, B, and E, $J$. Biol.Chem. 1985, 260, 10461-10466.

7. Black, J. D.; Dolly, J. O. Interaction of I-125 Labeled Botulinum Neurotoxins with NerveTerminals .2. Autoradiographic Evidence for Its Uptake into Motor Nerves by AcceptorMediated Endocytosis,J. Cell Biol. 1986, 103, 535-544.

8. Black, J. D.; Dolly, J. O. Interaction of I-125 Labeled Botulinum Neurotoxins with NerveTerminals .1. Ultrastructural Autoradiographic Localization and Quantitation of Distinct 
Membrane Acceptors for Type-a and Type-B on Motor Nerves, J. Cell Biol. 1986, 103, 521-534.

9. Pirazzini, M.; Azarnia Tehran, D.; Zanetti, G.; Megighian, A.; Scorzeto, M.; Fillo, S.; Shone, C. C.; Binz, T.; Rossetto, O.; Lista, F.; Montecucco, C. Thioredoxin and its reductase are present on synaptic vesicles, and their inhibition prevents the paralysis induced by botulinum neurotoxins, Cell Rep 2014, 8, 1870-1878.

10. Montecucco, C.; Schiavo, G. Structure and function of tetanus and botulinum neurotoxins, Quart. Rev. of Biophys. 1995, 28, 423-472.

11. Singh, B. R. Intimate details of the most poisonous poison, Nat. Struct. Biol. 2000, 7, 617619.

12. Binz, T.; Blasi, J.; Yamasaki, S.; Baumeister, A.; Link, E.; Sudhof, T. C.; Jahn, R.; Niemann, H. Proteolysis of SNAP-25 by types E and A botulinal neurotoxins, J. Biol. Chem. 1994, $269,1617-1620$.

13. Blasi, J.; Chapman, E. R.; Yamasaki, S.; Binz, T.; Niemann, H.; Jahn, R. Botulinum neurotoxin $\mathrm{C} 1$ blocks neurotransmitter release by means of cleaving HPC-1/syntaxin, EMBO J. 1993, 12, 4821-4828.

14. Schiavo, G.; Benfenati, F.; Poulain, B.; Rossetto, O.; Polverino de Laureto, P.; DasGupta, B. R.; Montecucco, C. Tetanus and botulinum-B neurotoxins block neurotransmitter release by proteolytic cleavage of synaptobrevin, Nature 1992, 359, 832-835.

15. Schiavo, G.; Rossetto, O.; Catsicas, S.; Polverino de Laureto, P.; DasGupta, B. R.; Benfenati, F.; Montecucco, C. Identification of the nerve terminal targets of botulinum neurotoxin serotypes A, D, and E, J. Biol. Chem. 1993, 268, 23784-23787. 
16. Schiavo, G.; Shone, C. C.; Rossetto, O.; Alexander, F. C.; Montecucco, C. Botulinum neurotoxin serotype F is a zinc endopeptidase specific for VAMP/synaptobrevin, J. Biol. Chem. 1993, 268, 11516-11519.

17. Schmidt, J. J.; Stafford, R. G.; Bostian, K. A. Type A botulinum neurotoxin proteolytic activity: development of competitive inhibitors and implications for substrate specificity at the S1' binding subsite, FEBS Lett 1998, 435, 61-64.

18. Schmidt, J. J.; Stafford, R. G. A high-affinity competitive inhibitor of type A botulinum neurotoxin protease activity, FEBS Lett 2002, 532, 423-426.

19. Silvaggi, N. R.; Wilson, D.; Tzipori, S.; Allen, K. N. Catalytic features of the botulinum neurotoxin A light chain revealed by high resolution structure of an inhibitory peptide complex, Biochemistry 2008, 47, 5736-5745.

20. Zuniga, J. E.; Schmidt, J. J.; Fenn, T.; Burnett, J. C.; Arac, D.; Gussio, R.; Stafford, R. G.; Badie, S. S.; Bavari, S.; Brunger, A. T. A potent peptidomimetic inhibitor of botulinum neurotoxin serotype A has a very different conformation than SNAP-25 substrate, Structure 2008, 16, 1588-1597.

21. Kumaran, D.; Rawat, R.; Ludivico, M. L.; Ahmed, S. A.; Swaminathan, S. Structure- and substrate-based inhibitor design for Clostridium botulinum neurotoxin serotype A, $J$. Biol. Chem.2008, 283, 18883-18891.

22. Hale, M.; Oyler, G.; Swaminathan, S.; Ahmed, S. A. Basic tetrapeptides as potent intracellular inhibitors of type A botulinum neurotoxin protease activity, J. Biol. Chem. 2011, 286, 1802-1811.

23. Kumar, G.; Kumaran, D.; Ahmed, S. A.; Swaminathan, S. Peptide inhibitors of botulinum neurotoxin serotype A: design, inhibition, cocrystal structures, structure-activity 
relationship and pharmacophore modeling, Acta Crystallogr. D Biol. Crystallogr. 2012, $68,511-520$.

24. Boldt, G. E.; Kennedy, J. P.; Janda, K. D. Identification of a potent botulinum neurotoxin a protease inhibitor using in situ lead identification chemistry, Org. Lett. 2006, 8, 17291732.

25. Silvaggi, N. R.; Boldt, G. E.; Hixon, M. S.; Kennedy, J. P.; Tzipori, S.; Janda, K. D.; Allen, K. N. Structures of Clostridium botulinum Neurotoxin Serotype A Light Chain complexed with small-molecule inhibitors highlight active-site flexibility, Chem. Biol. 2007, 14, 533-542.

26. Moe, S. T.; Thompson, A. B.; Smith, G. M.; Fredenburg, R. A.; Stein, R. L.; Jacobson, A. R. Botulinum neurotoxin serotype A inhibitors: small-molecule mercaptoacetamide analogs, Bioorg. Med. Chem. 2009, 17, 3072-3079.

27. Eubanks, L. M.; Hixon, M. S.; Jin, W.; Hong, S.; Clancy, C. M.; Tepp, W. H.; Baldwin, M. R.; Malizio, C. J.; Goodnough, M. C.; Barbieri, J. T.; Johnson, E. A.; Boger, D. L.; Dickerson, T. J.; Janda, K. D. An in vitro and in vivo disconnect uncovered through highthroughput identification of botulinum neurotoxin A antagonists, Proc. Natl. Acad. Sci. U. S. A. 2007, 104, 2602-2607.

28. Li, B.; Pai, R.; Cardinale, S. C.; Butler, M. M.; Peet, N. P.; Moir, D. T.; Bavari, S.; Bowlin, T. L. Synthesis and biological evaluation of botulinum neurotoxin a protease inhibitors, $J$. Med. Chem. 2010, 53, 2264-2276.

29. Silhar, P.; Eubanks, L. M.; Seki, H.; Pellet, S.; Javor, S.; Tepp, W. H.; Johnson, E. A.; Janda, K. D. Targeting Botulinum A Cellular Toxicity: A Prodrug Approach, J. Med. Chem. 2013, 56, 7870-7879. 
30. Caglic, D.; Krutein, M. C.; Bompiani, K. M.; Barlow, D. J.; Benoni, G.; Pelletier, J. C.; Reitz, A. B.; Lairson, L. L.; Houseknecht, K. L.; Smith, G. R.; Dickerson, T. J. Identification of Clinically Viable Quinolinol Inhibitors of Botulinum Neurotoxin A Light Chain, J. Med. Chem. 2014, 57, 669-676.

31. Stura, E. A.; Le Roux, L.; Guitot, K.; Garcia, S.; Bregant, S.; Beau, F.; Vera, L.; Collet, G.; Ptchelkine, D.; Bakirci, H.; Dive, V. Structural framework for covalent inhibition of Clostridium botulinum neurotoxin A by targeting Cys165, J. Biol. Chem. 2012, 287, $33607-33614$.

32. Kumar, G.; Swaminathan, S. Recent Developments with Metalloprotease Inhibitor Class of Drug Candidates for Botulinum Neurotoxins, Curr. Top. Med. Chem. 2015.

33. Anne, C.; Turcaud, S.; Quancard, J.; Teffo, F.; Meudal, H.; Fournie-Zaluski, M. C.; Roques, B. P. Development of potent inhibitors of botulinum neurotoxin type B, J. Med. Chem. 2003, 46, 4648-4656.

34. Anne, C.; Blommaert, A.; Turcaud, S.; Martin, A. S.; Meudal, H.; Roques, B. P. Thioderived disulfides as potent inhibitors of botulinum neurotoxin type B: implications for zinc interaction, Bioorg. Med. Chem. 2003, 11, 4655-4660.

35. Kumaran, D.; Eswaramoorthy, S.; Furey, W.; Navaza, J.; Sax, M.; Swaminathan, S. Domain organization in Clostridium botulinum neurotoxin type $\mathrm{E}$ is unique: its implication in faster translocation, J. Mol. Biol. 2009, 386, 233-245.

36. Wang, J.; Meng, J.; Lawrence, G. W.; Zurawski, T. H.; Sasse, A.; Bodeker, M. O.; Gilmore, M. A.; Fernandez-Salas, E.; Francis, J.; Steward, L. E.; Aoki, K. R.; Dolly, J. O. Novel chimeras of botulinum neurotoxins A and E unveil contributions from the binding, 
translocation, and protease domains to their functional characteristics, J. Biol. Chem. 2008, 283, 16993-17002.

37. Agarwal, R.; Eswaramoorthy, S.; Kumaran, D.; Binz, T.; Swaminathan, S. Structural analysis of botulinum neurotoxin type E catalytic domain and its mutant Glu212-->Gln reveals the pivotal role of the Glu212 carboxylate in the catalytic pathway, Biochemistry 2004, 43, 6637-6644.

38. Agarwal, R.; Swaminathan, S. SNAP-25 substrate peptide (residues 180-183) binds to but bypasses cleavage by catalytically active Clostridium botulinum neurotoxin E, J. Biol. Chem. 2008, 283, 25944-25951.

39. Kumar, G.; Agarwal, R.; Swaminathan, S. Discovery of a fluorene class of compounds as inhibitors of botulinum neurotoxin serotype E by virtual screening, Chem. Commun. 2012, 48, 2412-2414.

40. Group, C. C. (2011) Molecular Operating Environment, 2011.10 ed.

41. Mehler, E. L.; Solmajer, T. Electrostatic effects in proteins: comparison of dielectric and charge models, Protein Eng. 1991, 4, 903-910.

42. Burnett, J. C.; Li, B.; Pai, R.; Cardinale, S. C.; Butler, M. M.; Peet, N. P.; Moir, D.; Bavari, S.; Bowlin, T. Analysis of Botulinum Neurotoxin Serotype A Metalloprotease Inhibitors: Analogs of a Chemotype for Therapeutic Development in the Context of a Three-Zone Pharmacophore, Open Access Bioinformatics 2010, 2010, 11-18. 
${ }^{\star}$ Graphical Abstract (for review)

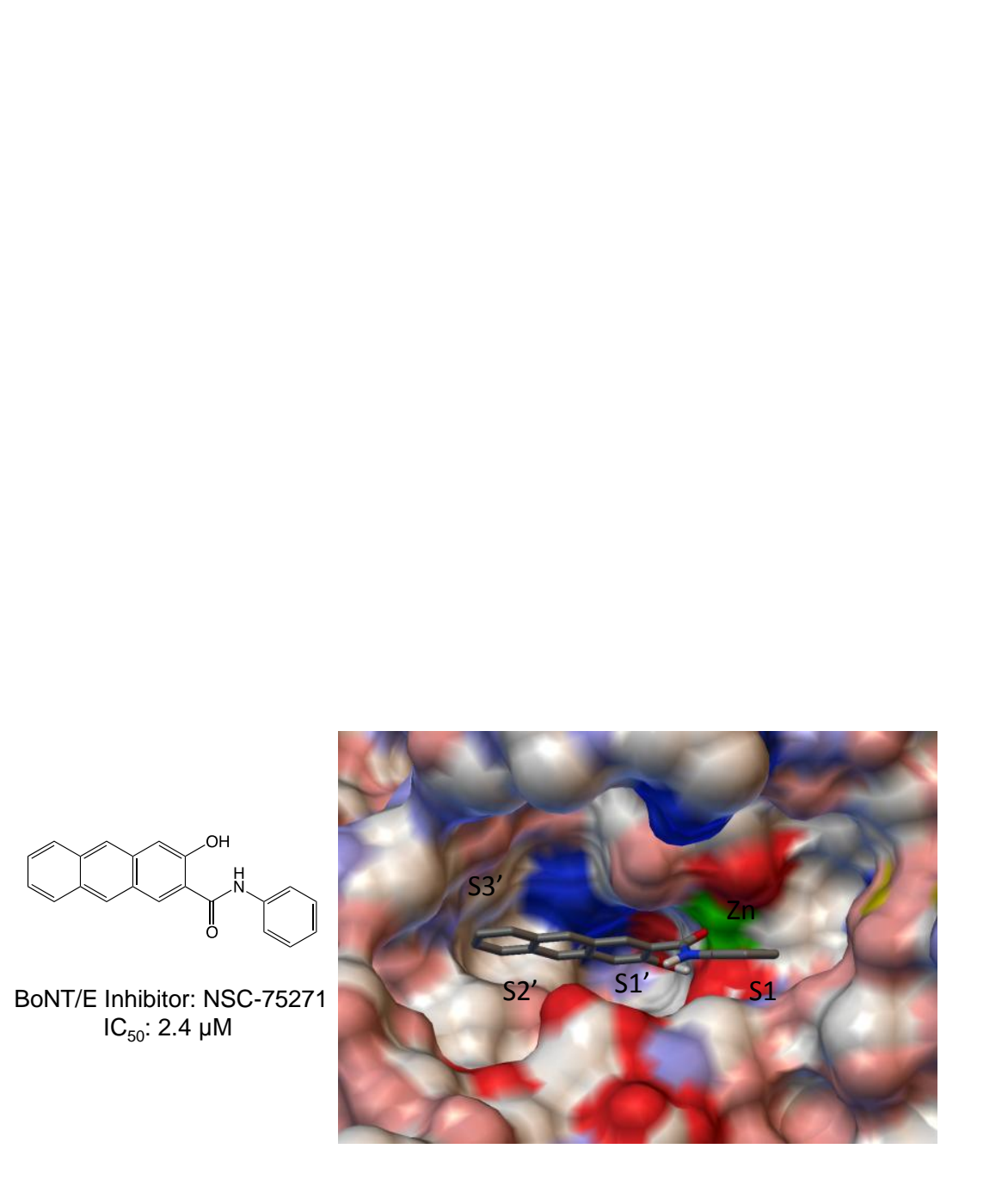

$\mathrm{IC}_{50}: 2.4 \mu \mathrm{M}$

BoNT/E Inhibitor: NSC-75271

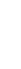

*Graphical Abstract (for review)

\section{)}

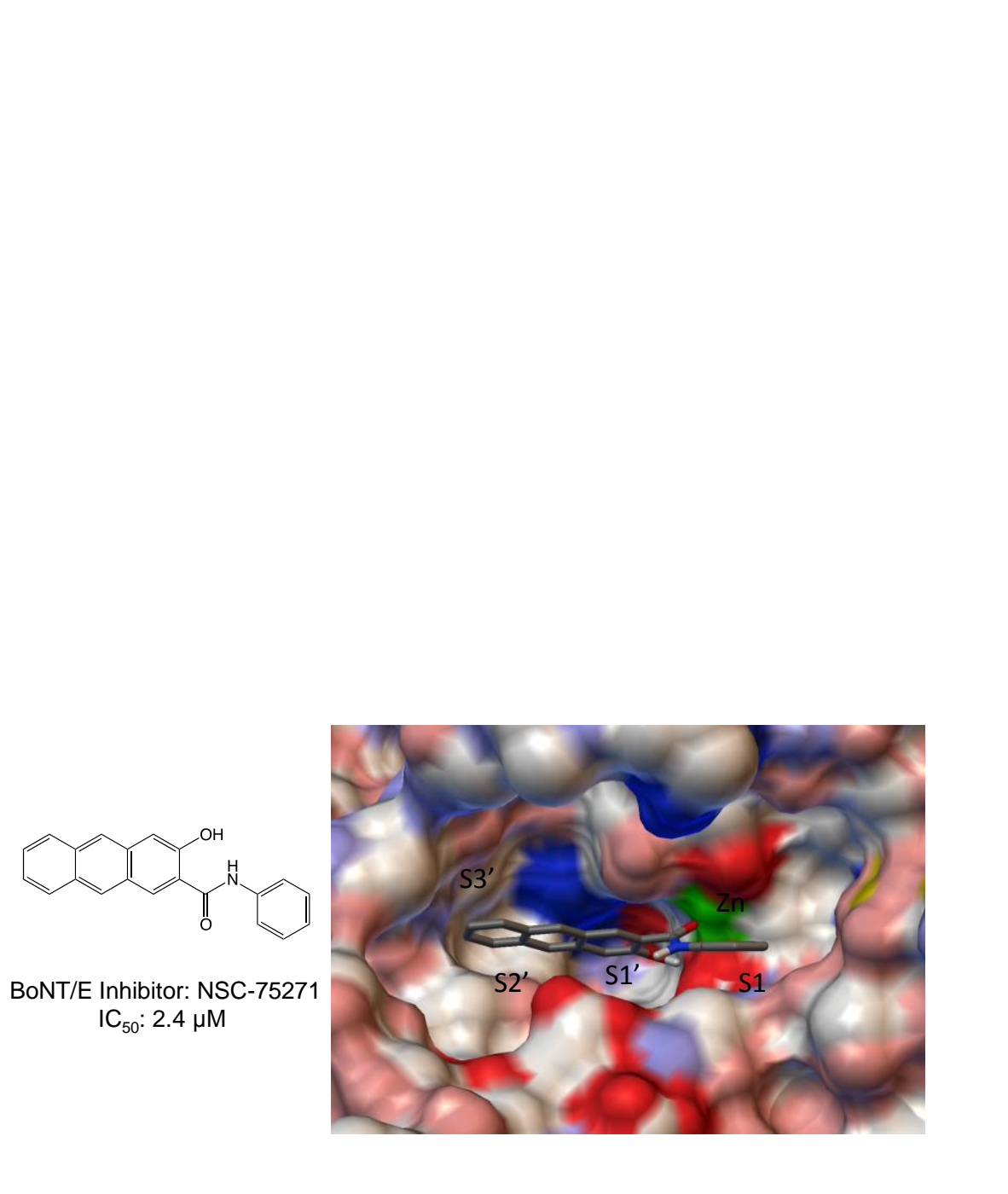

